\title{
Dynamics of localization in a waveguide
}

C.W.J. Beenakker

Instrtuut-Lorentz, Unvversitert Leiden

P.O. Box 9506, 2300 RA Leiden, The Netherlands

\begin{abstract}
This is a review of the dynamics of wave propagation through a disordered $N$-mode waveguide in the localized regime. The basic quantities considered are the Wigner-Smith and single-mode delay tımes, plus the time-dependent power spectrum of a reflected pulse. The long-time dynamics is dominated by resonant transmission over length scales much larger than the localization length. The corresponding distribution of the Wigner-Smith delay times is the Laguerre ensemble of random-matrix theory. In the power spectrum the resonances show up as a $t^{-2}$ tail after $N^{2}$ scattering times. In the distribution of single-mode delay times the resonances introduce a dynamic coherent backscattering effect, that provides a way to distinguish localization from absorption.
\end{abstract}

\section{Introduction}

Light localization, one of the two central themes of this meeting, has its roots in electron localization. Much of the theory was developed first for electrical conduction in metals at low temperatures, and then adapted to propagation of electromagnetic radiation through disordered dielectric media $[1,2]$. Low-temperature conduction translates into propagation that is monochromatic in the frequency domain, hence static in the time domain.

This historical reason may explain in part why much of the literature on localization of light deals exclusively with static properties. Of course one can think of other reasons, such as that a laser is a highly monochromatic light source. It is not accidental that one of the earliest papers on wave localization in the time domain [3] appeared in the context of seismology, where the natural wave source (an earthquake or explosion) is more appropriately described by a delta function in time than a delta function in frequency.

Our own interest in the dynamics of localization came from its potential as a diagnostic tool. The signature of static localization, an exponential decay of the transmitted intensity with distance, is not unique, since absorption gives an exponential decay as well [4]. This is at the origin of the difficulties surrounding an unambiguous demonstration of three-dimensional localization of light [5]. The dynamics of localization and absorption are, however, entirely different. One such dynamical signature of localization [6] is reviewed in this lecture. 

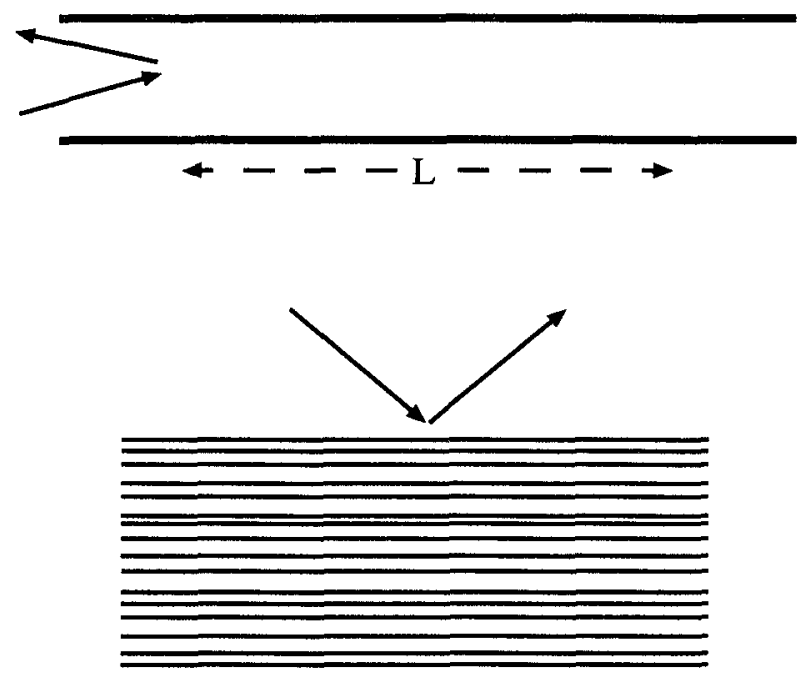

Figure 1. The top diagram shows the quasi-one-dimensional geometry considered in this review. The waveguide contains a region of length $L$ (dotted) with randomly located scatterers that reflects a wave incident from one end (arrows). The number of propagating modes $N$ may be arbitrarily large. The one-dimensional case $N=1$ is equivalent to the layered geometry shown in the bottom diagram. Each of the parallel layers is homogeneous but differs from the others by a random variation in composition and/or thickness.

Localization is a non-perturbative phenomenon and this severely complicates the theoretical problem. In two- and three-dimensional geometries (thin films or bulk materials) not even the static case has been solved completely [7]. The situation is more favorable in a onedimensional waveguide geometry, where a complete solution of static localization exists $[7,8]$. The introduction of dynamical aspects into the problem is a further complication, and we will therefore restrict ourselves to the waveguide geometry (see Fig. 1). The number $N$ of propagating modes in the waveguide may be arbitrarily large, so that the geometry is more appropriately called quast-one-dimensional. (The strictly one-dimensional case $N=1$ is equivalent to a layered material.)

The basic dynamical quantity that we will consider is the autocorrelator of the time-dependent wave amplitude $u(t)$,

$$
a_{\omega}(t)=\int_{-\infty}^{\infty} d t^{\prime} e^{-\imath \omega t^{\prime}} u(t) u\left(t+t^{\prime}\right)
$$

If the incident wave is a pulse in time, then the transmitted or reflected wave consists of rapid fluctuations with a slowly varying envelope (see Fig. 2). The correlator $a_{\omega}(t)$ selects the frequency component $\omega$ of 


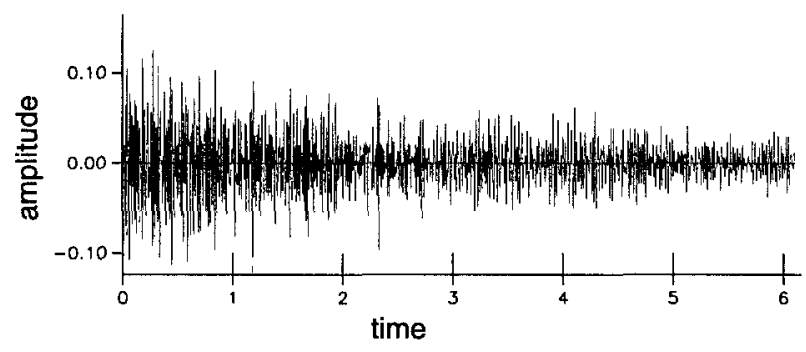

Figure 2. Computer simulation of an acoustic plane wave pulse reflected by a randomly layered medium. The medium is a model for the subsurface of the Earth, with a sound velocity that depends only on the depth. The figure shows the reflected wave amplitude as a function of time (arbitrary units). The incident pulse strikes the surface at time zero. From Ref. [9].

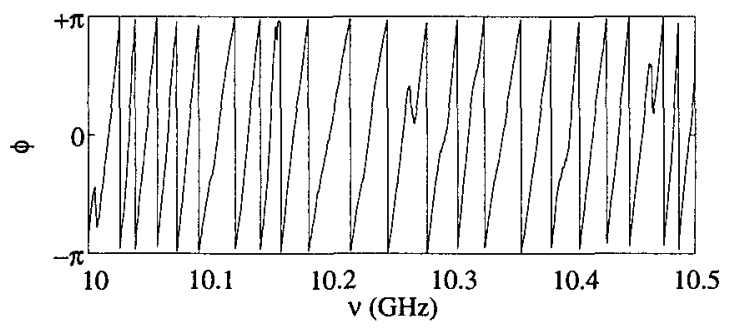

Figure 3. Frequency dependence of the phase (modulo $2 \pi$ ) of microwave radiation transmitted through a disordered waveguide. The waveguide consists of a $1 \mathrm{~m}$ long, $7.6 \mathrm{~cm}$ diameter copper tube containing randomly positioned polystyrene spheres (1.27 $\mathrm{cm}$ diameter, $0.52 \%$ volume filling fraction). Wire antennas are used as the emitter and detector at the two ends of the tube. From Ref. [10].

the rapid fluctuations. The remaining $t$-dependence is governed by the propagation time through the waveguide.

If the incident wave is not a pulse in time but a narrow band in frequency, then it is more convenient to study the frequency correlator

$$
a_{\omega}(\delta \omega)=\int_{-\infty}^{\infty} d t e^{i \delta \omega t} a_{\omega}(t)=u^{*}(\omega) u(\omega+\delta \omega)
$$

The Fourier transformed wave amplitude $u(\omega)=\int d t e^{i \omega t} u(t) \equiv I^{1 / 2} e^{i \phi}$ is complex, containing the real intensity $I(\omega)$ and phase $\phi(\omega)$. Most of the dynamical information is contained in the phase factor, which winds around the unit circle at a speed $d \phi / d \omega$ determined by the propagation time (see Fig. 3).

The correlator $a$ depends sensitively on the random locations of the scatterers in the waveguide, that give rise to the localization. This calls for a statistical treatment, in which we consider the probability distribution of $a$ in an ensemble of waveguides with different scatterer 
configurations. The method of random-matrix theory has proven to be very effective at obtaining statistical distributions for static scattering properties [8]. The extension to dynamical properties reviewed here is equally effective for studies of the reflected wave. The time dependence of the transmitted wave is more problematic, for reasons that we will discuss.

\section{Low-frequency dynamics}

The low-frequency regime is most relevant for optical and microwave experiments $[4,10,11]$, where one usually works with an incident beam that has a narrow frequency bandwidth relative to the inverse propagation time through the system. We assume that the length $L$ of the waveguide is long compared to the (static) localization length $\xi=N l$, which is equal to the product of the number of propagating modes $N$ and the mean free path $l$. The reflected wave amplitudes $r_{m n}$ in mode $m$ (for unit incident wave amplitude in mode $n$ ) are contained in an $N \times N$ reflection matrix $r$. This matrix is unitary, provided we can disregard absorption in the waveguide. It is also symmetric, because of reciprocity. (We do not consider the case that time-reversal symmetry is broken by some magneto-optical effect.)

The correlator

$$
C_{\omega}(\delta \omega)=r^{\dagger}(\omega) r(\omega+\delta \omega)
$$

is the product of two unitary matrices, so it is also unitary. Its eigenvalues $\exp \left(i \phi_{n}\right), n=1,2, \ldots N$, contain the phase shifts $\phi_{n}$. Since $\phi_{n} \equiv 0$ for all $n$ if $\delta \omega=0$, the relevant dynamical quantity at low frequencies is the limit

$$
\tau_{n}=\lim _{\delta \omega \rightarrow 0} \frac{\phi_{n}}{\delta \omega}
$$

which has the dimension of a time. It is known as the Wigner-Smith delay time, after the authors who first studied it in the context of nuclear scattering $[12,13]$. The $\tau_{n}$ 's may equivalently be defined as the eigenvalues of the Hermitian time-delay matrix $Q$,

$$
Q(\omega)=-i r^{\dagger} \frac{d r}{d \omega}=U^{\dagger} \operatorname{diag}\left(\tau_{1}, \tau_{2}, \ldots \tau_{N}\right) U
$$

Experiments typically measure not the product of matrices, as in Eq. (3), but the product of amplitudes, as in Eq. (2). The amplitude measured within a single speckle (or coherence area) corresponds to a single matrix element. The typical observable is therefore not the Wigner-Smith delay time but a different dynamical quantity called the 
single-channel (or single-mode) delay time $[10,11]$ :

$$
\tau_{m n}=\lim _{\delta \omega \rightarrow 0} \operatorname{Im} \frac{r_{m n}^{*}(\omega) r_{m n}(\omega+\delta \omega)}{\delta \omega\left|r_{m n}(\omega)\right|^{2}} .
$$

If we decompose the complex reflection amplitude into intensity and phase, $r_{m n}=I^{1 / 2} e^{\imath \phi}$, then the single-channel delay time is the phase derivative, $\tau_{m n}=d \phi / d \omega \equiv \phi^{\prime}$. Since the reflection matrix $r(\omega+\delta \omega)$ has for small $\delta \omega$ the expansion

$$
r_{m n}(\omega+\delta \omega)=\sum_{k} U_{k m} U_{k n}\left(1+i \tau_{k} \delta \omega\right)
$$

we can write the single-channel delay time as a linear combination of the Wigner-Smith times,

$$
\tau_{m n} \equiv \phi^{\prime}=\operatorname{Re} \frac{A_{1}}{A_{0}}, A_{k}=\sum_{\imath} \tau_{\imath}^{k} U_{\imath m} U_{\imath n}
$$

We will consider separately the probability distribution of these two dynamical quantities, following Refs. $[6,14]$.

\subsection{WigNER-SMITH DELAY TIME}

There is a close relationship between dynamic scattering problems without absorption and static problems with absorption [15]. Physically, this relationship is based on the notion that absorption acts as a "counter" for the delay time of a wave packet [16]. Mathematically, it is based on the analyticity of the scattering matrix in the upper half of the complex plane. Absorption with a spatially uniform rate $1 / \tau_{\mathrm{a}}$ is equivalent to a shift in frequency by an imaginary amount $\delta \omega=\mathrm{i} / 2 \tau_{\mathrm{a}} .{ }^{1}$ If we denote the reflection matrix with absorption by $r\left(\omega, \tau_{\mathrm{a}}\right)$, then $r\left(\omega, \tau_{\mathrm{a}}\right)=r\left(\omega+\mathrm{i} / 2 \tau_{\mathrm{a}}\right)$. For weak absorption we can expand

$$
r\left(\omega+\mathrm{i} / 2 \tau_{\mathrm{a}}\right) \approx r(\omega)+\frac{\mathrm{i}}{2 \tau_{\mathrm{a}}} \frac{d}{d \omega} r(\omega)=r(\omega)\left[1-\frac{1}{2 \tau_{\mathrm{a}}} Q(\omega)\right] .
$$

As before, we have assumed that transmission can be neglected so that $r$ is unitary and $Q$ is Hermitian. Eq. (9) implies that the matrix product

1 To see this, note that absorption is represented by a positive imaginary part of the dielectric constant $\varepsilon=1+i / \omega \tau_{\mathrm{a}}$ (for $\omega \tau_{\mathrm{a}} \gg 1$ ). Since $\varepsilon$ is multiplied by $\omega^{2}$ in the wave equation, a small imaginary increment $\omega \rightarrow \omega+i \delta \omega$ is equivalent to absorption with rate $2 \delta \omega$. In the presence of a fluctuating real part of $\varepsilon$, an imaginary shift in frequency will lead to a spatially fluctuating absorption rate, but this is statistically equivalent to homogeneous absorption with an increased scattering rate. 
$\mathrm{rr}^{\dagger}$ for weak absorption is related to the time-delay matrix $Q$ by a unitary transformation [14],

$$
r\left(\omega, \tau_{\mathrm{a}}\right) r^{\dagger}\left(\omega, \tau_{\mathrm{a}}\right)=r(\omega)\left[1-\frac{1}{\tau_{\mathrm{a}}} Q(\omega)\right] r^{\dagger}(\omega)
$$

The eigenvalues $R_{1}, R_{2}, \ldots R_{N}$ of $r r^{\dagger}$ in an absorbing medium are real numbers between 0 and 1, called the reflection eigenvalues. Because a unitary transformation leaves the eigenvalues unchanged, one has $R_{n}=1-\tau_{n} / \tau_{a}$. This relationship between reflection eigenvalues and Wigner-Smith delay times is useful because the effects of absorption have received more attention in the literature than dynamic effects. In particular, the case of a single-mode disordered waveguide with absorption was solved as early as 1959 , in the course of a radio-engineering problem [17]. The multi-mode case was solved more recently $[18,19]$. The distribution is given by the Laguerre ensemble, after a transformation of variables from $R_{n}$ to $\lambda_{n}=R_{n}\left(1-R_{n}\right)^{-1}$ :

$$
P\left(\left\{\lambda_{n}\right\}\right) \propto \prod_{i<j}\left|\lambda_{i}-\lambda_{j}\right|^{\beta} \prod_{k} \exp \left[-\left(\alpha \tau_{\mathrm{s}} / \tau_{\mathrm{a}}\right)(\beta N+2-\beta) \lambda_{k}\right] .
$$

Here $\tau_{\mathrm{s}}$ is the scattering time of the disorder and $\alpha$ is a numerical coefficient of order unity. ${ }^{2}$ The symmetry index $\beta=1$ in the presence of time-reversal symmetry. (The case $\beta=2$ of broken time-reversal symmetry is rarely realized in optics.) The eigenvalue density is given by a sum over Laguerre polynomials, hence the name "Laguerre ensemble" [20].

The relationship between the reflection eigenvalues for weak absorption and the Wigner-Smith delay times implies that the $\tau_{n}$ 's are distributed according to Eq. (11) if one substitutes $\lambda_{n} / \tau_{\mathrm{a}} \rightarrow 1 / \tau_{n}$ (since $\lambda_{n} \rightarrow\left(1-R_{n}\right)^{-1}$ for weak absorption). In terms of the rates $\mu_{n}=1 / \tau_{n}$ one has $[14]$

$$
P\left(\left\{\mu_{n}\right\}\right) \propto \prod_{i<j}\left|\mu_{i}-\mu_{j}\right|^{\beta} \prod_{k} \exp \left[-\gamma(\beta N+2-\beta) \mu_{k}\right] .
$$

${ }^{2}$ The coefficient $\alpha$ depends weakly on $N$ and on the dimensionality of the scattering: $\alpha=2$ for $N=1$; for $N \rightarrow \infty$ it increases to $\pi^{2} / 4$ or $8 / 3$ depending on whether the scattering is two or three-dimensional. The mean free path $l$, that we will encounter later on, is defined as $l=\alpha^{\prime} c \tau_{\mathrm{s}}$, with $\alpha^{\prime}=2$ for $N=1$ and $\alpha^{\prime} \rightarrow \pi / 2$ or $4 / 3$, respectively, for $N \rightarrow \infty$ in two or three dimensions. (The wave velocity is denoted by $c$.) Finally, the diffusion coefficient $D=c^{2} \tau_{\mathrm{s}} / d$ with $d=1$ for $N=1$ and $d \rightarrow 2$ or 3 for $N \rightarrow \infty$. The dimensionality that determines these coefficients is a property of the scattering. It is distinct from the dimensionality of the geometry. For example, a waveguide geometry (length much greater than width) is one-dimensional, but it may have $d=3$ (as in the experiments of Ref. [11]) or $d=2$ (as in the computer simulations of Ref. [6]). 
We have abbreviated $\gamma=\alpha \tau_{\mathrm{s}}$. For $N=1$ it is a simple $\beta$-independent exponential distribution $[21,22,23]$, or in terms of the original variable $\tau$

$$
P(\tau)=2 \gamma \tau^{-2} \exp (-2 \gamma / \tau)
$$

The slow $\tau^{-2}$ decay gives a logarithmically diverging mean delay time. The finite localization length $\xi$ is not sufficient to constrain the delay time, because of resonant transmission. Resonant states may penetrate arbitrarily far into the waveguide, and although these states are rare, they dominate the mean (and higher moments) of the delay time. The divergence is cut off for any finite length $L$ of the waveguide. Still, as long as $L \gg \xi$, the resonant states cause large sample-to-sample fluctuations of the delay times. These large fluctuations drastically modify the distribution of the single-channel delay time, as we will discuss next.

\subsection{Single-Channel Delay time}

In view of the relation (8), we can compute the distribution of the single-channel delay time $\phi^{\prime}$ from that of the Wigner-Smith delay times, if we also know the distribution of the matrix of eigenvectors $U$. For a disordered medium it is a good approximation to assume that $U$ is uniformly distributed in the unitary group, independent of the $\tau_{n}$ 's. The distribution $P\left(\phi^{\prime}\right)$ may be calculated analytically in the regime $N \gg 1$, which is experimentally relevant $(N \simeq 100$ in the microwave experiments of Ref. [11]).

In the large- $N$ limit the matrix elements $U_{m n}$ become independent complex Gaussian random numbers, with zero mean and variance $\left\langle\left|U_{m n}\right|^{2}\right\rangle=1 / N$. Since Eq. (8) contains the elements $U_{i m}$ and $U_{i n}$, we should distinguish between $n=m$ and $n \neq m$. Let us discuss first the case $n \neq m$ of different incident and detected modes. The average over the $U_{i n}$ 's amounts to doing a set of Gaussian integrations, with the result [6]

$$
P\left(\phi^{\prime}\right)=\left\langle\frac{1}{2}\left(B_{2}-B_{1}^{2}\right)\left(B_{2}+\phi^{2}-2 B_{1} \phi^{\prime}\right)^{-3 / 2}\right\rangle .
$$

The average $\langle\cdots\rangle$ is over the two spectral moments $B_{1}$ and $B_{2}$, defined by $B_{k}=\sum_{i} \tau_{i}^{k}\left|U_{i m}\right|^{2}$. The joint distribution $P\left(B_{1}, B_{2}\right)$, needed to perform the average, has a rather complicated form, for which we refer to Ref. [6].

The result (14) applies to the localized regime $L \gg \xi$. In the diffusive regime $l \ll L \ll \xi$ one has instead $[11,24]$

$$
P\left(\phi^{\prime}\right)=\left(Q / 2 \bar{\phi}^{\prime}\right)\left[Q+\left(\phi^{\prime} / \bar{\phi}^{\prime}-1\right)^{2}\right]^{-3 / 2} .
$$



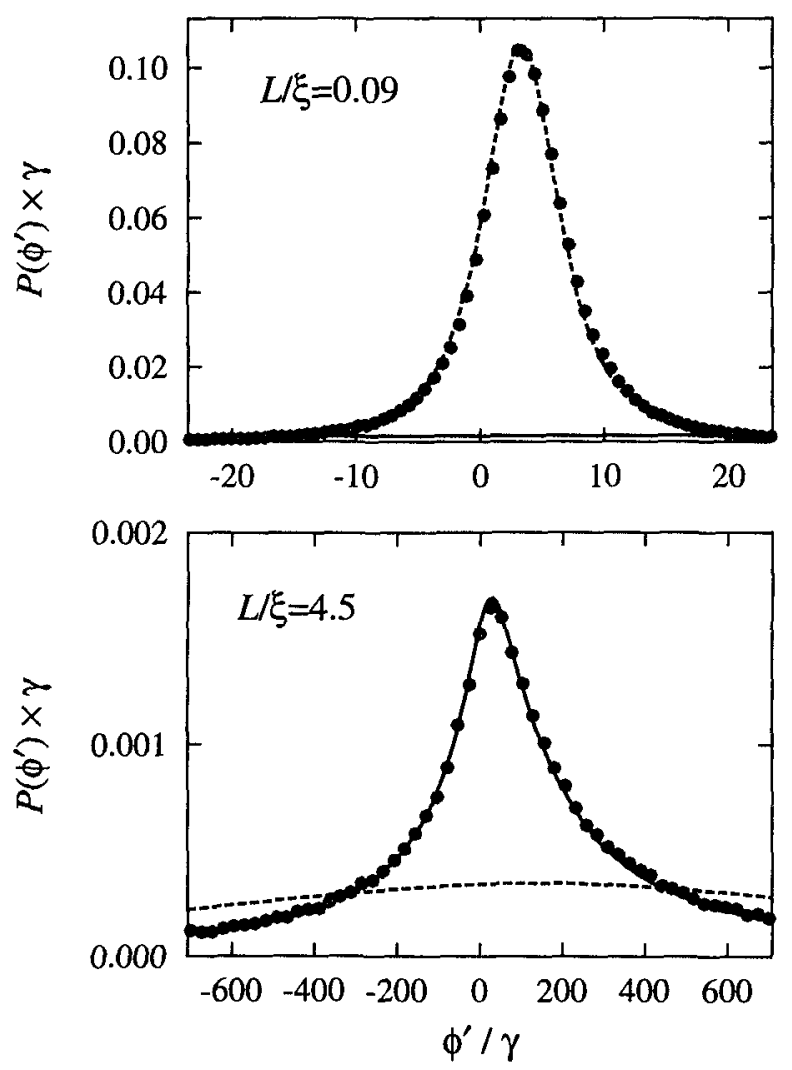

Figure 4. Distribution of the single-channel delay time $\phi^{\prime}$ in the diffusive regime (top panel) and localized regime (bottom panel). The results of numerical simulations (data points) are compared to the predictions (14) (solid curve) and (15) (dashed). These are results for different incident and detected modes $n \neq m$. From Ref. [6].

The constants are given by $Q \simeq L / l$ and $\bar{\phi}^{\prime} \simeq L / c$ up to numerical coefficients of order unity. Comparison of Eqs. (14) and (15) shows that the two distributions would be identical if statistical fluctuations in $B_{1}$ and $B_{2}$ could be ignored. However, as a consequence of the large fluctuations of the Wigner-Smith delay times in the localized regime, the distribution $P\left(B_{1}, B_{2}\right)$ is very broad and fluctuations have a substantial effect.

This is illustrated in Fig. 4, where we compare $P\left(\phi^{\prime}\right)$ in the two regimes. The data points are obtained from a numerical solution of the wave equation on a two-dimensional lattice, in a waveguide geometry with $N=50$ propagating modes. They agree very well with the analytical curves. The distribution (15) in the diffusive regime decays $\propto\left|\phi^{\prime}\right|^{-3}$, so that the mean delay time is finite (equal to $\bar{\phi}^{\prime}$ ). The distribution in the localized regime decays more slowly, $\propto\left|\phi^{\prime}\right|^{-2}$. 

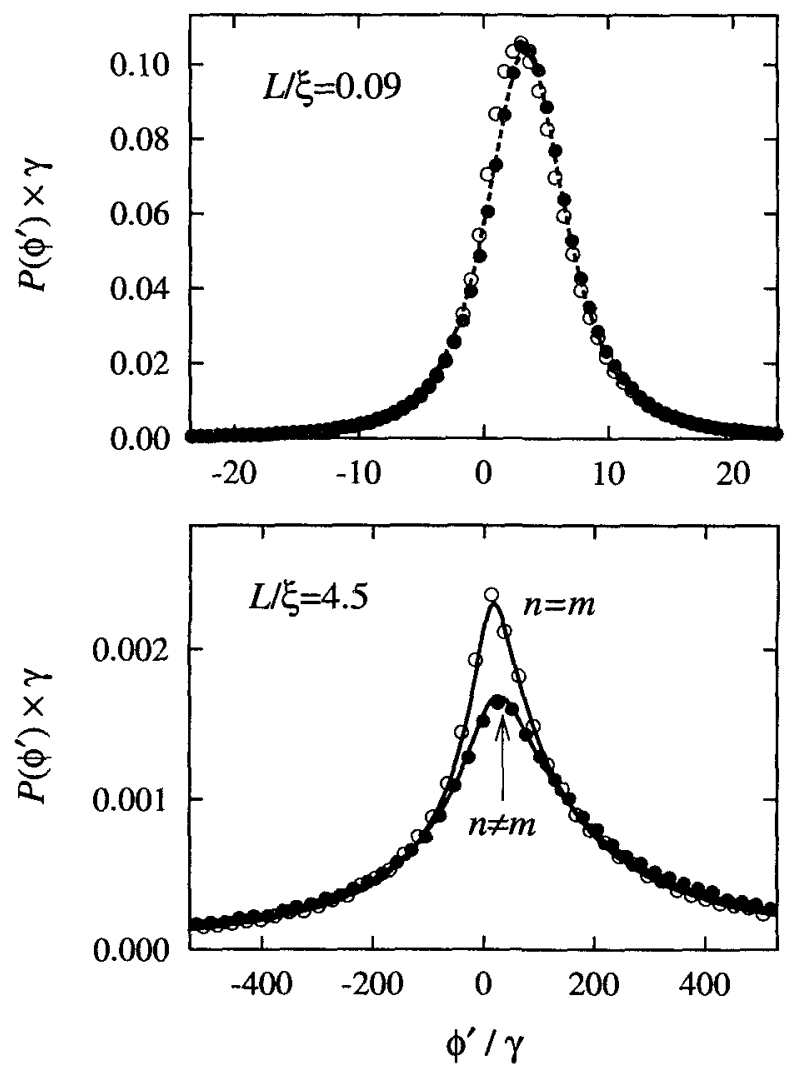

Figure 5. Same as the previous figure, but now comparing the case $n \neq m$ of different incident and detected modes (solid circles) with the equal-mode case $n=m$ (open circles). A coherent backscattering effect appears, but only in the localized regime. From Ref. [6].

The resulting logarithmic divergence of the mean delay time is cut off in the simulations by the finiteness of the waveguide length.

Notice that, although the most probable value of the single-channel delay time is positive, the tail of the distribution extends both to positive and negative values of $\phi^{\prime}$. This is in contrast to the WignerSmith delay time $\tau_{n}$, which takes on only positive values. The adjective "delay" in the name single-channel delay time should therefore not be taken literally. The difficulties in identifying the phase derivative with the duration of a scattering process have been emphasized by Büttiker [25].

We now turn to the case $n=m$ of equal-mode excitation and detection. An interesting effect of coherent backscattering appears in the localized regime, as shown in Fig. 5. The maximal value of $P\left(\phi^{\prime}\right)$ for $n=m$ is larger than for $n \neq m$ by a factor close to $\sqrt{2}$. (The precise 
value in the limit $N \rightarrow \infty$ is $\sqrt{2} \times \frac{4096}{1371 \pi}$.) In the diffusive regime, however, there is no difference in the distributions of the single-channel delay time for $n=m$ and $n \neq m$.

Coherent backscattering in the original sense is a static scattering property $[26,27]$. The distribution $P(I)$ of the reflected intensity differs if the detected mode is the same as the incident mode or not. The difference amounts to a rescaling of the distribution by a factor of two,

$$
P(I)=\left\{\begin{array}{cc}
N e^{-N I} & \text { if } n \neq m, \\
\frac{1}{2} N e^{-N I / 2} & \text { if } n=m,
\end{array}\right.
$$

so that the mean reflected intensity $\bar{I}$ becomes twice as large near the angle of incidence. It doesn't matter for this static coherent backscattering effect whether $L$ is large or small compared to $\xi$. The dynamic coherent backscattering effect, in contrast, requires localization for its existence, appearing only if $L>\xi$. This is the dynamical signature of localization mentioned in the introduction.

\subsection{TRANSMISSION}

Experiments on the delay-time distribution have so far only been carried out in transmission, not yet in reflection. The distribution (15) in the diffusive regime applies both to transmission and to reflection, only the constants $Q$ and $\bar{\phi}^{\prime}$ differ [24]. (In transmission, $Q$ is of order unity while $\bar{\phi}^{\prime} \simeq L^{2} / l c$.) Good agreement between theory and experiment has been obtained both with microwaves [11] and with light [4]. The microwave data is reproduced in Fig. 6. Absorption can not be neglected in this experiment ( $L$ exceeds the absorption length $l_{\mathrm{a}}$ by a factor 2.5 ), but this can be accounted for simply by a change in $Q$ and $\bar{\phi}^{\prime}$. The localization length is larger than $L$ by a factor of 5 , so that the system is well in the diffusive regime. It would be of interest to extend these experiments into the localized regime, both in transmission and in reflection. This would require a substantial reduction in absorption, to ensure that $L<\xi<l_{\mathrm{a}}$.

Theoretically, much less is known about the delay-time distribution in transmission than in reflection. While we have a complete theory in reflection, as described in the previous subsection, in transmission not even the $N=1$ case has been solved completely. Regardless of the value of $N$, one would expect $P\left(\phi^{\prime}\right)$ for $L \gg \xi$ to have the same $1 / \phi^{2}$ tail in transmission as it has in reflection, since in both cases the same resonances allow the wave to penetrate deeply into the localized region. For $N=1$ this is borne out by numerical simulations by BoltonHeaton et al. [28]. These authors also used a picture of one-dimensional resonant transport through localized states to study the decay of the 


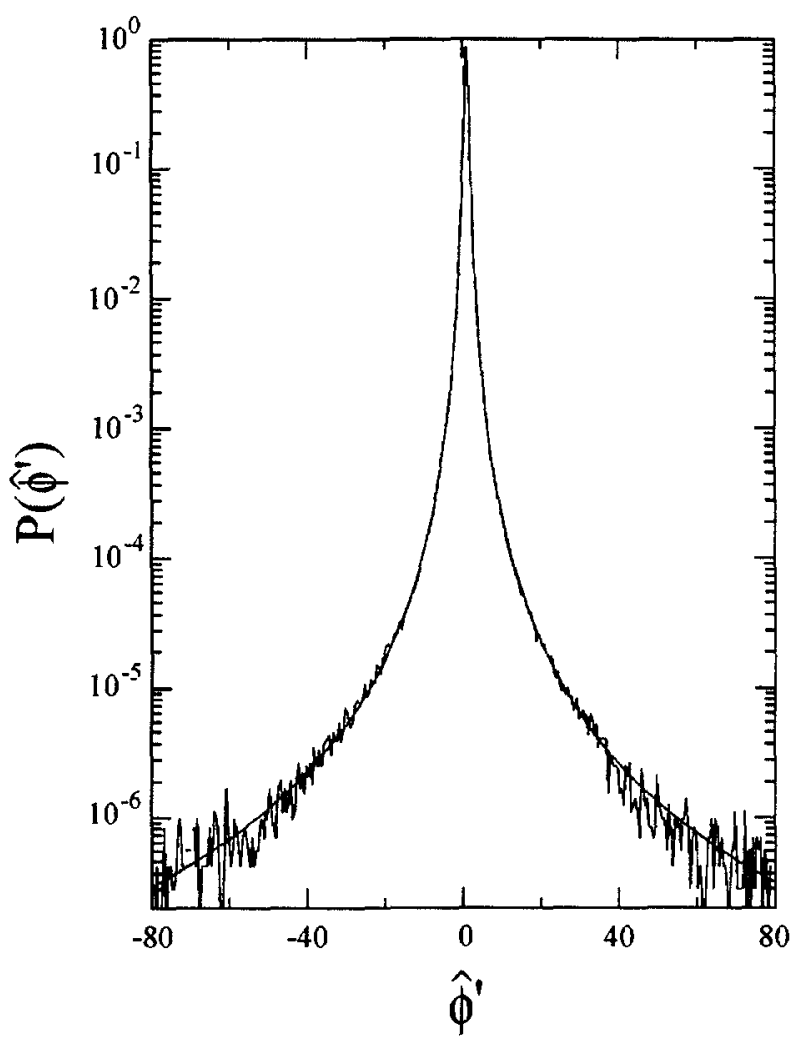

Figure 6. Distribution of the rescaled single-channel delay time $\hat{\phi}^{\prime}=\phi^{\prime} / \bar{\phi}^{\prime}$, measured in transmission at a frequency $\nu \equiv \omega / 2 \pi=18.1 \mathrm{GHz}$ on the system described in Fig. 3. The smooth curve through the data is the analytical prediction (15) of diffusion theory (with $Q=0.31$ ). From Ref. [11].

weighted delay time $I \phi^{\prime}$ (with $I$ the transmitted intensity). They found an algebraic decay for $P\left(I \phi^{\prime}\right)$, just as for $P\left(\phi^{\prime}\right)$, but with a different exponent $-4 / 3$ instead of -2 . It is not known how this carries over to $N>1$.

Because of the finite length $L$ of the waveguide, these algebraic tails are only an intermediate asymptotics. For $N=1$ and exponentially large times $\left|\phi^{\prime}\right|>\tau_{\mathrm{s}} e^{L / l}$ the delay-time distribution has the more rapid decay $[28,29]$

$$
P\left(\phi^{\prime}\right) \propto \exp \left[-(l / L) \ln ^{2}\left(\phi^{\prime} / \tau_{\mathrm{s}}\right)\right]
$$

Such a log-normal tail is likely to exist in the multi-mode case as well, but this has so far only been demonstrated in the diffusive regime $l \ll L \ll \xi[30,31,32]$. The $1 / \phi^{2}$ intermediate asymptotics does not appear in that regime. 


\section{High-frequency dynamics}

\subsection{REFLECTION}

The high-frequency limit of the correlator (3) of the reflection matrices is rather trivial. The two matrices $r^{\dagger}(\omega)$ and $r(\omega+\delta \omega)$ become uncorrelated for $\delta \omega \rightarrow \infty$, so that $C$ becomes the product of two independent random matrices. The distribution of each of these matrices may be regarded as uniform in the unitary group, and then $C$ is also uniformly distributed. This is the circular ensemble of random-matrix theory [20], so called because the eigenvalues $\exp \left(i \phi_{n}\right)$ are spread out uniformly along the unit circle. Their joint distribution is

$$
P\left(\left\{\phi_{n}\right\}\right) \propto \prod_{n<m}\left|e^{i \phi_{n}}-e^{i \phi_{m}}\right|^{\beta} .
$$

This distribution contains no dynamical information.

\subsection{TRANSMISSION}

The transmission problem is more interesting at high frequencies. Let us consider the ensemble-averaged correlator of the transmission matrix elements

$$
\left\langle a_{\omega}(\delta \omega)\right\rangle=\left\langle t_{m n}^{*}(\omega) t_{m n}(\omega+\delta \omega)\right\rangle .
$$

Following Ref. [33], we proceed as we did in Sec. 2.1, by mapping the dynamic problem without absorption onto a static problem with absorption.

We make use of the analyticity of the transmission amplitude $t_{m n}(\omega+$ $i y$ ), at complex frequency $\omega+i y$ with $y>0$, and of the symmetry relation $t_{m n}(\omega+i y)=t_{m n}^{*}(-\omega+i y)$. The product of transmission amplitudes $t_{m n}(\omega+z) t_{m n}(-\omega+z)$ is an analytic function of $z$ in the upper half of the complex plane. If we take $z$ real, equal to $\frac{1}{2} \delta \omega$, we obtain the product $t_{m n}\left(\omega+\frac{1}{2} \delta \omega\right) t_{m n}^{*}\left(\omega-\frac{1}{2} \delta \omega\right)$ in Eq. (19) (the difference with $t_{m n}(\omega+\delta \omega) t_{m n}^{*}(\omega)$ being statistically irrelevant for $\left.\delta \omega \ll \omega\right)$. If we take $z$ imaginary, equal to $i / 2 \tau_{\mathrm{a}}$, we obtain the transmission probability $T=\left|t_{m n}\left(\omega+i / 2 \tau_{\mathrm{a}}\right)\right|^{2}$ at frequency $\omega$ and absorption rate $1 / \tau_{\mathrm{a}}$. We conclude that the ensemble average of $a$ can be obtained from the ensemble average of $T$ by analytic continuation to imaginary absorption rate:

$$
\left\langle a_{\omega}(\delta \omega)\right\rangle=\langle T\rangle \text { for } 1 / \tau_{\mathbf{a}} \rightarrow-i \delta \omega .
$$

Higher moments of $a$ are related to higher moments of $T$ by $\left\langle a^{p}\right\rangle=$ $\left\langle T^{p}\right\rangle$ for $1 / \tau_{\mathrm{a}} \rightarrow-i \delta \omega$. Unfortunately, this is not sufficient to determine the entire probability distribution $P(a)$, because moments of the 
form $\left\langle a^{p} a^{* q}\right\rangle$ can not be obtained by analytic continuation. This is a complication of the transmission problem. The reflection problem is simpler, because the (approximate) unitarity of the reflection matrix $r$ provides additional information on the distribution of the correlator of the reflection amplitudes. This explains why in Sec. 2.1 we could use the mapping between the dynamic and absorbing problems to calculate the entire distribution function of the eigenvalues of $r^{\dagger}(\omega) r(\omega+\delta \omega)$ in the limit $\delta \omega \rightarrow 0$.

We will apply the mapping first to the single-mode case $(N=1)$ and then to the case $N \gg 1$ of a multi-mode waveguide.

\subsubsection{One mode}

The absorbing problem for $N=1$ was solved by Freilikher, Pustilnik, and Yurkevich [34]. Applying the mapping (20) to their result we find ${ }^{3}$

$$
\left\langle a_{\omega}(\delta \omega)\right\rangle=\exp (i \delta \omega L / c-L / l),
$$

in the regime $c / l \ll \delta \omega \ll\left(\omega^{2} c / l\right)^{1 / 3}$. (The high-frequency cutoff is due to the breakdown of the random-phase approximation [35].) The absolute value $|\langle a\rangle|=\exp (-L / l)$ is $\delta \omega$-independent in this regime. For $L \ll l$ one has ballistic motion, hence $\langle a\rangle=\exp (i \delta \omega L / c)$ is simply a phase factor, with the ballistic time of flight $L / c$. Comparing with Eq. (21) we see that localization does not change the frequency dependence of the correlator for large $\delta \omega$, which remains given by the ballistic time scale, but only introduces a frequency-independent weight factor.

The implication of this result in the time domain is that $\left\langle a_{\omega}(t)\right\rangle$ has a peak with weight $\exp (-L / l)$ at the ballistic time $t=L / c$. Such a ballistic peak is expected for the propagation of classical particles through a random medium, but it is surprising to find that it applies to wave dynamics as well.

\subsubsection{Many modes}

Something similar happens for $N \gg 1$. The transmission probability in an absorbing multi-mode waveguide was calculated by Brouwer [36],

$$
\langle T\rangle=\frac{l}{N \xi_{\mathrm{a}} \sinh \left(L / \xi_{\mathrm{a}}\right)} \exp \left(-\frac{L}{2 N l}\right),
$$

for absorption lengths $\xi_{\mathrm{a}}=\sqrt{D \tau_{\mathrm{a}}}$ in the range $l \ll \xi_{\mathrm{a}} \ll \xi$. The length $L$ of the waveguide should be $\gg l$, but the relative magnitude of $L$ and

${ }^{3}$ The coefficient in front of the factor $L / l$ in the exponent in Eq. (21) would be $-\frac{1}{3}$ according to the results of Ref. [34]. This would disagree with numerical simulations, which clearly indicate $|\langle a\rangle|=\exp (-L / l)$ (K. J. H. van Bemmel, unpublished). The error can be traced back to Eq. (39) in Ref. [34]. 
$\xi$ is arbitrary. Substitution of $1 / \tau_{\mathrm{a}}$ by $-i \delta \omega$ gives the correlator

$$
\left\langle a_{\omega}(\delta \omega)\right\rangle=\frac{l \sqrt{-i \tau_{D} \delta \omega}}{N L \sinh \sqrt{-i \tau_{D} \delta \omega}} \exp \left(-\frac{L}{2 N l}\right),
$$

where $\tau_{D}=L^{2} / D$ is the diffusion time. The range of validity of Eq. (23) is $L / \xi \ll \sqrt{\tau_{D} \delta \omega} \ll L / l$, or equivalently $D / \xi^{2} \ll \delta \omega \ll c / l$. In the diffusive regime, for $L \ll \xi$, the correlator (23) reduces to the known result [37] from perturbation theory.

For $\max \left(D / L^{2}, D / \xi^{2}\right) \ll \delta \omega \ll c / l$ the decay of the absolute value of the correlator is a stretched exponential,

$$
\left|\left\langle a_{\omega}(\delta \omega)\right\rangle\right|=\frac{2 l}{N L} \sqrt{\tau_{D} \delta \omega} \exp \left(-\sqrt{\frac{1}{2} \tau_{D} \delta \omega}-\frac{L}{2 N l}\right) .
$$

In the localized regime, when $\xi$ becomes smaller than $L$, the onset of this tail is pushed to higher frequencies, but it retains its functional form. The weight of the tail is reduced by a factor $\exp (-L / 2 N l)$ in the presence of time-reversal symmetry. (There is no reduction factor if time-reversal symmetry is broken [33].)

In Fig. 7 we compare the results of numerical simulations in a twodimensional waveguide geometry with the analytical high-frequency prediction. We see that the correlators for different values of $L / \xi$ converge for large $\delta \omega$ to a curve that lies somewhat above the theoretical prediction. The offset is probably due to the fact that $N$ is not $\gg 1$ in the simulation. Regardless of this offset, the simulation confirms both analytical predictions: The stretched exponential decay $\propto$ $\exp \left(-\sqrt{\tau_{D} \delta \omega / 2}\right)$ and the exponential suppression factor $\exp (-L / 2 \xi)$. We emphasize that the time constant $\tau_{D}=L^{2} / D$ of the high-frequency decay is the diffusion time for the entire length $L$ of the waveguide even though the localization length $\xi$ is up to a factor of 12 smaller than $L$.

We can summarize these findings [33] for the single-mode and multimode waveguides by the statement that the correlator of the transmission amplitudes factorises in the high-frequency regime: $\left\langle a_{\omega}(\delta \omega)\right\rangle \rightarrow$ $f_{1}(\delta \omega) f_{2}(\xi)$. The frequency dependence of $f_{1}$ depends on the diffusive time through the waveguide, even if it is longer than the localization length. Localization has no effect on $f_{1}$, but only on $f_{2}$.

\section{Propagation of a pulse}

If the incident wave is a short pulse, then the separation into low- and high-frequency dynamics is less natural. Ideally one would like to know 


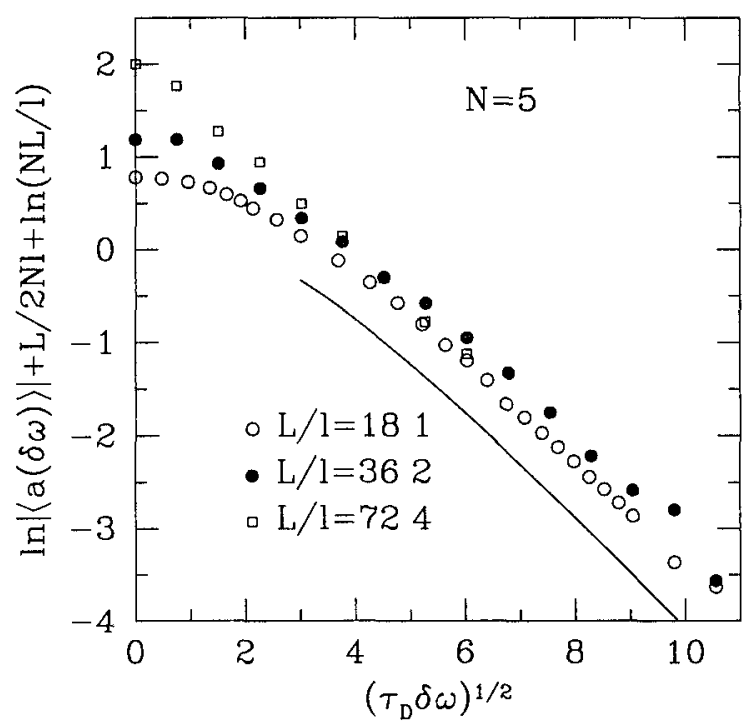

Figure 7 Frequency dependence of the logarithm of the absolute value of the correlator $\left\langle a_{\omega}(\delta \omega)\right\rangle$ The data points follow from a numerical simulation for $N=5$, the solid curve is the analytical high-frequency result (24) for $N \gg 1$ The decay of the correlator is given by the diffusive time constant $\tau_{D}=L^{2} / D$ even if the length $L$ of the waveguide is greater than the localization length $\xi=6 l$ The offset of about 06 between the numerical and analytıcal results is probably a finite- $N$ effect From Ref [33]

the entire time dependence of the correlator $a_{\omega}(t)$ introduced in Eq. (1). A complete solution exists [38] for the ensemble-averaged correlator in the case of reflection,

$$
\begin{aligned}
\left\langle a_{\omega}(t)\right\rangle & =\int_{-\infty}^{\infty} \frac{d \delta \omega}{2 \pi} e^{-\imath \delta \omega t}\left\langle r_{m n}^{*}(\omega) r_{m n}(\omega+\delta \omega)\right\rangle \\
& =\frac{1+\delta_{m n}}{N(N+1)} \int_{-\infty}^{\infty} \frac{d \delta \omega}{2 \pi} e^{-\imath \delta \omega t}\left\langle\operatorname{Tr} C_{\omega}(\delta \omega)\right\rangle .
\end{aligned}
$$

The second equality follows from the representation

$$
r(\omega \pm \delta \omega / 2)=U^{\mathrm{T}} e^{ \pm \imath \Phi / 2} U,
$$

with $\Phi=\operatorname{diag}\left(\phi_{1}, \phi_{2}, \ldots \phi_{N}\right)$ a diagonal matrix and $U$ uniformly distributed in the unitary group. The factor $1+\delta_{m n}$ is due to coherent backscattering. It is convenient to work with the normalized power spectrum,

$$
\mathcal{P}_{\omega}(t)=\frac{N+1}{1+\delta_{m n}}\left\langle a_{\omega}(t)\right\rangle=\frac{1}{N} \int_{-\infty}^{\infty} \frac{d \delta \omega}{2 \pi} e^{-\imath \delta \omega t}\left\langle\operatorname{Tr} C_{\omega}(\delta \omega)\right\rangle
$$


normalized such that $\int_{0}^{\infty} d t \mathcal{P}_{\omega}(t)=1$.

Since $e^{i \phi_{n}}$ is an eigenvalue of the unitary matrix $C$, one can write

$$
\left\langle\operatorname{Tr} C_{\omega}(\delta \omega)\right\rangle=\int_{0}^{2 \pi} d \phi \rho(\phi) e^{i \phi}
$$

where $\rho(\phi)=\left\langle\sum_{n=1}^{N} \delta\left(\phi-\phi_{n}\right)\right\rangle$ is the phase-shift density. This density can be obtained from the corresponding density $\rho(R)$ of reflection eigenvalues $R_{n}$ (eigenvalues of $r r^{\dagger}$ ) in an absorbing medium, by analytic continuation to imaginary absorption rate: $i / \tau_{a} \rightarrow \delta \omega, R_{n} \rightarrow \exp \left(i \phi_{n}\right)$. The densities are related by

$$
\rho(\phi)=\frac{N}{2 \pi}+\frac{1}{\pi} \operatorname{Re} \sum_{n=1}^{\infty} e^{-i n \phi} \int_{0}^{1} R^{n} \rho(R) d R,
$$

as one can verify by equating moments. This is a quick and easy way to solve the problem, since the probability distribution of the reflection eigenvalues is known $[18,19]$ : it is given by the Laguerre ensemble (11). The density $\rho(R)$ can be obtained from that as a series of Laguerre polynomials, using methods from random-matrix theory [20]. Eq. (29) then directly gives the density $\rho(\phi)$.

One might wonder whether one could generalize Eq. (29) to reconstruct the entire distribution function $P\left(\left\{\phi_{n}\right\}\right)$ from the Laguerre ensemble of the $R_{n}$ 's. The answer is no, unless $\delta \omega$ is infinitesimally small (as in Sec. 2.1). The reason that the method of analytic continuation can not be used to obtain correlations between the $\phi_{n}$ 's is that averages of negative powers of $\exp \left(i \phi_{n}\right)$ are not analytic in the reflection eigenvalues. For example, for the two-point correlation function one would need to know the average $\left\langle\exp \left(i \phi_{n}-i \phi_{m}\right)\right\rangle \rightarrow\left\langle R_{n} R_{m}^{-1}\right\rangle$ that diverges in the absorbing problem. It is possible to compute $P\left(\left\{\phi_{n}\right\}\right)$ for any $\delta \omega-$ but that requires a different approach, for which we refer to Ref. [38].

The calculation of the power spectrum from Eqs. (27)--(29) is easiest in the absence of time-reversal symmetry, because $\rho(R)$ then has a particularly simple form. One obtains the power spectrum [38]

$$
\begin{aligned}
\mathcal{P}_{\omega}(t) & =-\frac{1}{N} \frac{d}{d t} F(t / 2 N \gamma), \\
F(t) & =\frac{1}{t+1} \sum_{n=0}^{N-1}\left(\frac{t-1}{t+1}\right)^{n} P_{n}\left(\frac{t^{2}+1}{t^{2}-1}\right),
\end{aligned}
$$

where $P_{n}$ is a Legendre polynomial. (Recall that $\gamma=\alpha \tau_{\mathrm{s}}$, cf. Sec. 2.1.) In the single-mode case Eq. (30) simplifies to [3]

$$
\mathcal{P}_{\omega}(t)=2 \gamma(t+2 \gamma)^{-2}
$$




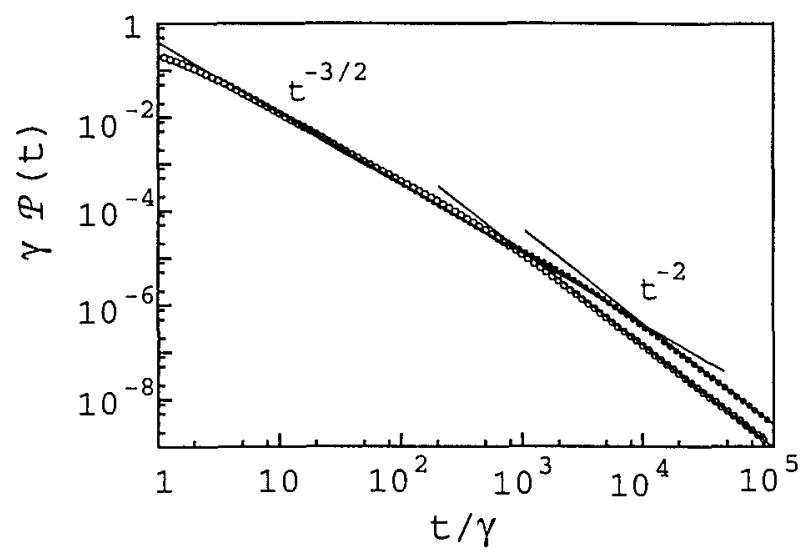

Figure 8. Time dependence of the power spectrum of a reflected pulse in the absence of time-reversal symmetry, calculated from Eq. (30) for $N=7$ (open circles) and $N=21$ (filled circles). The intermediate-time asymptote $\propto t^{-3 / 2}$ and the large-time asymptote $\propto t^{-2}$ are shown as straight lines in this double-logarithmic plot. The prefactor is $N$-independent for intermediate times but $\propto N$ for large times (notice the relative offset of the large-time asymptotes). Courtesy of $M$. Titov.

It decays as $t^{-2}$. For $N \rightarrow \infty$ Eq. (30) simplifies to

$$
\mathcal{P}_{\omega}(t)=t^{-1} \exp (-t / \gamma) I_{1}(t / \gamma)
$$

where $I_{1}$ is a modified Bessel function. The power spectrum now decays as $t^{-3 / 2}$. For any finite $N$ we find a crossover from $\mathcal{P}=\sqrt{\gamma / 2 \pi} t^{-3 / 2}$ for $\tau_{\mathrm{s}} \ll t \ll N^{2} \tau_{\mathrm{s}}$ to $\mathcal{P}=2 N \gamma t^{-2}$ for $t \gg N^{2} \tau_{\mathrm{s}}$. This is illustrated in Fig. 8.

In the presence of time-reversal symmetry the exact expression for $\mathcal{P}_{\omega}(t)$ is more cumbersome but the asymptotics carries over with minor modifications. In particular, the large- $N$ limit (33) with its $t^{-3 / 2}$ decay remains the same, while the $t^{-2}$ decay changes only in the prefactor: $\mathcal{P}=(N+1) \gamma t^{-2}$ for $t \gg N^{2} \tau_{\mathrm{s}}$.

The quadratic tail of the time-dependent power spectrum of a pulse reflected from an infinitely long waveguide is the same as the quadratic tail of the delay-time distribution that we have encountered in Sec. 2. It is natural to assume that the power spectrum for transmission through a localized waveguide of finite length has the same quadratic decay, with a cross-over to a log-normal tail for exponentially large times, cf. Sec. 2.3. 


\section{Conclusion}

We now have a rather complete picture of the dynamics of a wave reflected by a disordered waveguide. The dynamical information is contained in the phase factors $e^{i \phi_{n}}$ that are the eigenvalues of the product of reflection matrices $r^{\dagger}(\omega) r(\omega+\delta \omega)$. Three regimes can be distinguished, depending on the magnitude of the length scale $l_{\delta \omega}=\sqrt{D / \delta \omega}$ associated with the frequency difference $\delta \omega$ :

- Ballistic regime, $l_{\delta \omega}<l$. This is the high-frequency regime. The statistics of the $\phi_{n}$ 's is given by the circular ensemble, Eq. (18).

- Localized regime, $l_{\delta \omega}>\xi$. This is the low-frequency regime. The $\phi_{n}$ 's are now distributed according to the Laguerre ensemble (12).

- Diffusive regime, $l<l_{\delta \omega}<\xi$. The distribution of the $\phi_{n}$ 's does not belong to any of the known ensembles of random-matrix theory [38].

The emphasis in this review has been on the localized regime. The dynamics is then dominated by resonances that allow the wave to penetrate deep into the waveguide. Such resonances correspond to large delay times $\tau_{n}=\lim _{\delta \omega \rightarrow 0} \phi_{n} / \delta \omega$. The distribution of the largest delay time $\tau_{\max }$ follows from the distribution of the smallest eigenvalue in the Laguerre ensemble [39]. For $\beta=1$ it is given by

$$
P\left(\tau_{\max }\right)=\gamma N(N+1) \tau_{\max }^{-2} \exp \left(-\gamma N(N+1) / \tau_{\max }\right) .
$$

It has a long-time tail $\propto 1 / \tau_{\max }^{2}$, so that the mean delay time diverges (in the limit of an infinitely long waveguide). A subtle and unexpected consequence of the resonances is the appearance of a dynamic coherent backscattering effect in the distribution of the single-mode delay times. Unlike the conventional coherent backscattering effect in the static intensity, the dynamic effect requires localization for its existence. The recent progress in time-resolved measurements of light scattering from random media, reported at this meeting [4], should enable observation of this effect.

Extension of the theory to two- and three-dimensional localization remains a challenging problem for future research. We believe that the dynamic coherent backscattering effect will persist in higher dimensions, provided the localization length remains large compared to the mean free path. Several methods have been proposed to distinguish absorption from localization in the static intensity [40, 41]. The effect reviewed here could provide this information from a different, dynamic, perspective. 


\section{Acknowledgements}

It is a pleasure to acknowledge the fruitful collaboration on this topic with K. J. H. van Bemmel, P. W. Brouwer, H. Schomerus, and M. Titov. This research was supported by the "Nederlandse organisatie voor Wetenschappelijk Onderzoek" (NWO) and by the "Stichting voor Fundamenteel Onderzoek der Materie" (FOM).

\section{References}

1. Scattering and Localization of Classical Waves in Random Media, edited by P. Sheng (World Scientific, Singapore, 1990).

2. B. A. van Tiggelen, in Diffuse Waves in Complex Media, edited by J.-P. Fouque, NATO Science Series C531 (Kluwer, Dordrecht, 1999).

3. B. White, P. Sheng, Z. Q. Zhang, and G. Papanicolaou, Phys. Rev. Lett. 59, 1918 (1987).

4. A. Lagendijk, J. Gómez Rivas, A. Imhof, F. J. P. Schuurmans, and R. Sprik, in this volume.

5. F. Scheffold, R. Lenke, R. Tweer, and G. Maret, Nature 398, 206 (1999); D. S. Wiersma, J. Gómez Rivas, P. Bartolini, A. Lagendijk, and R. Righini, Nature 398, 207 (1999).

6. H. Schomerus, K. J. H. van Bemmel, and C. W. J. Beenakker, cond-mat/0004049; cond-mat/0009014.

7. K. Efetov, Supersymmetry in Disorder and Chaos (Cambridge University, Cambridge, 1997).

8. C. W. J. Beenakker, Rev. Mod. Phys. 69, 731 (1997).

9. M. Asch, W. Kohler, G. Papanicolaou, M. Postel, and B. White, SIAM Review 33, 519 (1991).

10. P. Sebbah, O. Legrand, and A. Z. Genack, Phys. Rev. E 59, 2406 (1999).

11. A. Z. Genack, P. Sebbah, M. Stoytchev, and B. A. van Tiggelen, Phys. Rev. Lett. 82, 715 (1999).

12. E. P. Wigner, Phys. Rev. 98, 145 (1955).

13. F. T. Smith, Phys. Rev. 118, 349 (1960).

14. C. W. J. Beenakker and P. W. Brouwer, cond-mat/9908325.

15. V. I. Klyatskin and A. I. Saichev, Usp. Fiz. Nauk 162, 161 (1992) [Sov. Phys. Usp. 35, 231 (1992)].

16. S. A. Ramakrishna and N. Kumar, Phys. Rev. B 61, 3163 (2000).

17. M. E. Gertsenshtein and V. B. Vasil'ev, Teor. Veroyatn. Primen. 4, 424 (1959); 5, 3(E) (1960) [Theor. Probab. Appl. 4, 391 (1959); 5, 340(E) (1960)].

18. C. W. J. Beenakker, J. C. J. Paasschens, and P. W. Brouwer, Phys. Rev. Lett. 76, 1368 (1996).

19. N. A. Bruce and J. T. Chalker, J. Phys. A 29, 3761 (1996); 29, 6681(E) (1996).

20. M. L. Mehta, Random Matrices (Academic, New York, 1991).

21. A. M. Jayannavar, G. V. Vijayagovindan, and N. Kumar, Z. Phys. B 75, 77 (1989).

22. J. Heinrichs, J. Phys. Condens. Matter 2, 1559 (1990).

23. A. Comtet and C. Texier, J. Phys. A 30, 8017 (1997). 
24. B. A. van Tiggelen, P. Sebbah, M. Stoytchev, and A. Z. Genack, Phys. Rev. E 59, 7166 (1999).

25. M. Büttiker, in Electronıc Properties of Multılayers and Low-Dimensıonal Semiconductor Structures, edited by J. M. Chamberlain, L. Eaves, and J. C. Portal, NATO ASI Series B231 (Plenum, New York, 1990).

26. M. P. van Albada and A. Lagendijk, Phys. Rev. Lett. 55, 2692 (1985).

27. P.-E. Wolf and G. Maret, Phys. Rev. Lett. 55, 2696 (1985).

28. C. J. Bolton-Heaton, C. J. Lambert, V. I. Fal'ko, V. Prigodin, and A. J. Epstein, Phys. Rev. B 60, 10569 (1999).

29. B. L. Altshuler and V. N. Prigodin, Pis'ma Zh. Eksp. Fiz. 47, 36 (1988) [JETP Lett. 47, 43 (1988)].

30. B. L. Altshuler, V. E. Kravtsov, and I. V. Lerner, in Mesoscopıc Phenomena in Solvds, edited by B. L. Altshuler, P. A. Lee, and R. A. Webb (North-Holland, Amsterdam, 1991).

31. B. A. Muzykantskii and D. E. Khmelnitskii, Phys. Rev. B 51, 5480 (1995).

32. A. D. Mirlin, Phys. Rep. 326, 259 (2000).

33. C. W. J. Beenakker, K. J. H. van Bemmel, and P. W. Brouwer, Phys Rev. E 60, R6313 (1999).

34. V. Freilikher, M. Pustilnik, and I. Yurkevich, Phys. Rev. B 50, 6017 (1994).

35. V. Freilikher and M. Pustilnik, Phys. Rev. B 55, R653 (1997).

36. P. W. Brouwer, Phys. Rev. B 57, 10526 (1998).

37. R. Berkovits and S. Feng, Phys. Rep. 238, 135 (1994).

38. M. Titov and C. W. J. Beenakker, cond-mat/0005042.

39. A. Edelman, Lin. Alg. Appl. 159, 55 (1991).

40. A. A. Chabanov, M Stoytchev, and A. Z. Genack, Nature 404, 850 (2000).

41. B. A. van Tiggelen, A. Lagendijk, and D. S. Wiersma, Phys. Rev. Lett. 84, $4333(2000)$. 
